\title{
Rederivation of transgenic mice from iPS cells derived from frozen tissue
}

\author{
Angus Yiu-Fai Lee $\cdot$ K. C. Kent Lloyd
}

Received: 21 January 2010/Accepted: 25 March 2010/Published online: 16 April 2010

(C) The Author(s) 2010. This article is published with open access at Springerlink.com

\begin{abstract}
In mice, induced pluripotent stem (iPS) cells with embryonic stem (ES)-like characteristics have been derived by ectopic expression of four transcription factors in somatic cells: Sox2, Oct3/4, Klf4 and/or c-Myc. To date, iPS cells have only be made from freshly harvested tissues and cells. However, if iPS cells could be derived from frozen tissues and cells, then cryopreservation of tissues such as mouse tails could conceivably become a reliable alternative to the more traditional formats, like germplasm and ES cells, for the archiving of genetically altered mouse lines. To test this hypothesis, we sought to demonstrate that a live transgenic mouse line could be recovered from transgenic iPS cells derived from cryopreserved mouse tissues. Tails and tail-derived fibroblasts from a DsRED transgenic mouse were cryopreserved in the presence of $5 \%$ dimethylsulfoxide (DMSO) in liquid nitrogen for 1 week and 1 month, respectively. Afterward, tissues and cells were thawed and underwent nuclear reprogramming by molecular transfection to derive iPS cells which generated germline confirmed transgenic
\end{abstract}

A. Y.-F. Lee · K. C. Kent Lloyd $(\bowtie)$

Mouse Biology Program, Center for Comparative

Medicine, School of Veterinary Medicine, University of

California, 2795 2nd Street, Suite 400, Davis, CA 95618,

USA

e-mail: kclloyd@ucdavis.edu;

kclloyd@mail.compmed.ucdavis.edu

A. Y.-F. Lee

e-mail: ayflee@ucdavis.edu mice. Our results demonstrate for the first time that iPS cells can be efficiently derived from frozenstored-thawed tail tissue and fibroblasts and used to re-establish a transgenic mouse line. Therefore, this study provides conclusive evidence that, as a practical matter, frozen tails and fibroblasts can be used as an effective and reliable alternative to frozen germplasm and ES cells for the storage, maintenance, and distribution of genetically-altered mutant mice.

Keywords Cryopreservation - Reprogramming Pluripotent stem cells · Germline

\section{Introduction}

Until recently, it was thought that only embryonic stem (ES) cells derived from the inner cell mass of 3.5 days post coitus (dpc) blastocysts retained sufficient pluripotency to propagate indefinitely in vitro (Evan and Kaufman 1981; Martin 1981) and undergo targeted differentiation into a broad variety of cell types (Rathjen and Rathjen 2001; Boheler et al. 2002). Now, however, it is well established that induced pluripotent stem (iPS) cells with similar characteristics to ES cells can be derived from adult somatic cells by the introduction of four transcription factors, namely Oct3/4, sox2, c-myc and Klf4 (Takahashi and Yamanaka 2006; Wernig et al. 2007). This method allows the production of ES-like 
cells from any cell type without the need to manipulate or destroy an embryo. Since these initial achievements, we now know that a similar degree of pluripotentiality can be attained using only three of these transcription factors (Oct3/4, Sox 2 and Klf4) by omitting c-myc, which has been shown to induce tumor formation in iPS cell-derived animals (Okita et al. 2007; Nakagawa et al. 2007; Wernig et al. 2008). Using this modified reprogramming approach, different somatic cell types, such as fibroblasts, hepatocytes and gastric epithelial cells, have been shown to give rise to iPS cells efficiently (Takahashi and Yamanaka 2006; Wernig et al. 2007; Aoi et al. 2008).

To date, iPS cells have been generated by the reprogramming of cells isolated from freshly harvested tissues. There are numerous occasions when it would be useful, if not essential, to be able to effectively and reliably derive iPS cells from frozenthawed tissues. For example, snips of mouse tail that are typically harvested to extract DNA for genotyping by polymerase chain reaction (PCR) can be collected and cryopreserved quickly and easily for storage. A tail snip could then be thawed, fibroblasts isolated, and iPS cells derived for future research use. This procedure has numerous applications. For operational needs and other efficiencies, the ability to delay the derivation of iPS cells using frozen tissues obviates the need to pursue reprogramming of fresh cells immediately after harvest. For example, lengthy experimentation and/or invasive analysis may be required to select the cohort of animals out of a larger group from which to derive iPS cells for study. Also, reprogramming of frozen tissues to derive iPS cells can be processed together in one experiment in order to minimize scientific variability and enhance experimental consistency compared to individual experiments performed multiple times on freshly harvested tissues.

The other potentially useful application of "delayed" iPS cell reprogramming would be for the archiving of genetically-altered mutant mouse lines. Today, most mouse repositories archive frozen germplasm as an alternative to storing and maintaining mice as live breeding colonies "on the shelf". Cryopreservation of embryos is considered the gold standard for maintaining a frozen archive of mutant mouse lines, but the process is lengthy, costly, and laborious. Cryopreserving sperm is simpler, faster, and cheaper, but some strains, notably C57BL/6 in widespread use today, may not be viable for in vitro fertilization (IVF) due to poor post-thaw recovery of sperm motility and would require the use of intracytoplasmic sperm injection (ICSI) to recover the mouse line. Frozen ES cells can be thawed and injected into blastocysts to generate chimeric mice, but germline transmission of a mutant allele is not guaranteed. Further, except for ovaries, whole frozen tissues and organs generally are not viable after thawing. Nonetheless, small sections of tissues, such as tail tips, and fibroblasts isolated from them, can be readily collected, quickly and easily frozen, and maintained in cryostorage for minimal cost. In addition, cryopreserved tissues could serve as a viable backup to other cryopreserved formats, and provide an abundant supply of somatic cells from which iPS cells could be derived. Further, frozen tissues are easily distributed to and shared among researcher laboratories. Unfortunately, there are no published studies establishing the effectiveness and efficiency of reprogramming frozen-thawed somatic tissues to derive iPS cells that can subsequently be microinjected into blastocysts to produce chimeric embryos that give rise to germline-competent live mice.

Therefore, the purpose of this study was to determine the feasibility of using frozen-storedthawed somatic cells and tissues as a means to archive and maintain mutant mouse lines for distribution. To test this, our first aim was to determine the efficiency of deriving viable iPS cells by retroviral transfection of frozen-stored-thawed fibroblasts with three transcription factors: Oct3/4, Sox 2 and Klf4. Our second aim was to compare the effectiveness of deriving iPS cells from frozen mouse tails and tailderived fibroblasts. Our third aim was to determine the reliability of using derived iPS cells to generate chimeric mice that transmit a exogenously introduced transgene through the germline to subsequent generations of mice.

\section{Materials and methods}

Preparation of tail fibroblasts

DsRED transgenic male mice at around 4 weeks old were purchased from Jackson laboratory. To prepare 
1 week frozen tail, tails $(4-5 \mathrm{~cm})$ were harvested and rinsed with PBS several times. Afterwards the superficial dermis was removed. The tails were cut into $1 \mathrm{~cm}$ pieces and were placed into cryogenic vials (polypropylene, Nunc, Denmark) containing freezing solution (PBS supplemented with 5\% DMSO). Groups of tails were kept in freezing solution at $-20^{\circ} \mathrm{C}$ until frozen and then at $-80^{\circ} \mathrm{C}$ overnight. The next day, frozen tail tissues were placed in liquid nitrogen for 1 week storage, after which they were thawed in a $37^{\circ} \mathrm{C}$ water bath until ice was fully melted. The tail was rinsed with fibroblast medium [DMEM medium (Invitrogen) supplemented with $10 \%$ fetal bovine serum (Hyclone), $2 \mathrm{mM}$ L-glutamine (Invitrogen), $1 \mathrm{mM}$ sodium pyruvate (Invitrogen), and $100 \mathrm{U}$ and $100 \mu \mathrm{g} \mathrm{ml}^{-1}$ of penicillin and streptomycin (Invitrogen). Tail pieces were placed in a six well plate and were incubated in fibroblast medium at $37^{\circ} \mathrm{C}$. After 5 days, tail pieces were discarded and the fibroblasts were continued in culture until they reached $90 \%$ confluency. Then $8 \times 10^{5}$ fibrobalsts were taken for retroviral infection.

To prepare 1 month frozen fibroblasts, fibroblasts derived from fresh tail were grown in a six well plate for 6-7 days. They were harvested with $0.05 \%$ trypsin (Invitrogen) and were plated onto $10 \mathrm{~cm}$ culture dish. After 3-4 days, fibroblasts were harvested with trypsin and were stored $\left(8 \times 10^{5}\right.$ cells/ vial) in freezing solution (fibroblast medium supplement with $20 \%$ fetal bovine serum plus $10 \%$ DMSO) and frozen and stored as previously described, except this group was stored for 1 month.

\section{Retroviral infection}

The retroviral vectors (pMXs-Sox2, pMXs-Oct3/4 and pMXs-Klf4) were obtained from Addgene (Cambridge, MA). They were introduced into Plat-E cells (Cell Biolabs, Inc.) using FuGENE 6 transfection reagent (Roche). Twenty-seven microlitre of transfection reagent was added to DMEM medium and incubated at room temperature for $5 \mathrm{~min}$. Nine microgram of retroviral vector was added to this transfection mixture for $30 \mathrm{~min}$ at room temperature. After incubation, the DNA mixture was added to the $8 \times 10^{6}$ Plat-E cells (a derivative of $293 \mathrm{~T}$ cell line for retrovirus packaging) in $10 \mathrm{~cm}$ culture dish. The Plat-E cells were incubated at $37^{\circ} \mathrm{C}$ with $5 \% \mathrm{CO}_{2}$ for overnight. The next day, the medium was discarded, and fresh medium was added for another overnight incubation. Fibroblasts were seeded at $8 \times 10^{5}$ cells in a $10 \mathrm{~cm}$ culture dish coated with mitomycin C-treated STO feeders. After overnight culture, the retrovirus containing medium was filtered through a $0.45 \mu \mathrm{m}$ cellulose acetate filter (ColeParmer), polybrene was added to this medium to achieve final concentration equal to $4 \mu \mathrm{g} / \mathrm{ml}$. Then, the retrovirus containing medium was transferred to the $10 \mathrm{~cm}$ culture dish containing fibroblasts for transfection. After overnight incubation, the medium was replaced with fresh medium. Three days later, regular LIF containing ES cell medium was used to culture fibroblasts until visible iPS clones had been formed at around 2-3 weeks.

\section{Examination of the iPS cells}

\section{Alkaline phosphatase staining}

iPS cells were placed in 24-well culture plates and grown till confluent. The cell medium was aspirated and the iPS cells were washed by $1 \times$ PBST (1XPBS containing $0.05 \%$ Tween-20) briefly and fixed in fixing solution (Cell Biolabs, Inc) for $2 \mathrm{~min}$. The fixing solution was discarded and the cells were washed with 1 XPBST twice. The Stem ${ }^{\mathrm{TM}}$ TAG AP staining solution (Cell Biolabs, Inc) was added to the cells for incubation at room temperature and protected from light. The desired brownish color on the cell surface could be observed in 1-2 $\mathrm{h}$ under the microscope if there was alkaline phosphatase activity.

\section{Immuno-staining analysis}

Cells were washed briefly with PBS and then fixed with $4 \%$ paraformaldehyde for $20 \mathrm{~min}$. Then, the cells were washed three times with PBS for $5 \mathrm{~min}$ each. $0.3 \% \mathrm{H}_{2} \mathrm{O}_{2}$ solution was added to the cells for quenching endogenous peroxidase activity for $30 \mathrm{~min}$ following by washing the cells three times with PBS for $5 \mathrm{~min}$ each. The cells were incubated with the blocking solution (10\% goat serum) for $30 \mathrm{~min}$ and then with the SSEA1 antibody (Abcam) in 1:300 dilution for $3 \mathrm{~h}$. Staining was performed using the Vectastain ABC kit (Vector Laboratory) according to the manufacturer's recommendations. 


\section{Chromosome counting}

Cells were cultured for 2-3 days for metaphase preparation before harvesting. Chromosomes were dispersed by incubation with KARYO MAX colcemid solution (Gibco) for $1 \mathrm{~h}, 0.56 \% \mathrm{KCl}$ for $5 \mathrm{~min}$ and re-suspended in methanol:glacial acetic acid (3:1) fixative solution. Cells were finally stained with VECTASHIELD Mounting Medium with DAPI (Vector Laboratory) on the slide and chromosomes were counted.

\section{Generation of chimeras and PCR genotyping}

Two iPS cell clones from each treated group (i.e., 1 week frozen tail and 1 month frozen fibroblasts) were taken for blastocyst microinjection. Chimeras were produced by injecting iPS cells into 30-48 blastocysts harvested from 4 week old Balb/C females. The injected blastocysts were transferred into the uterus of pseudopregnant CD1 females. The male chimeras with high iPS cell contribution were crossed with CD1 females to identify germline transmission of the DsRED transgenic allele. $F_{1}$ offspring were examined for germline transmission by their coat color and were confirmed by PCR amplification of DsRED transgene using the following primers: DsRED-F (5'-CCCCGTAATGCAGA AGAAGA- $3^{\prime}$ ) and DsRED-R (5'-GGTGATGTCCA GCTTGGAGT- $3^{\prime}$ ) and by DsRED expression.

\section{Results}

Generation of iPS cells from frozen tail tissue and frozen fibroblasts

We reprogrammed fibroblasts isolated from tail snips that were harvested from 4 week old DsRED transgenic mice (Vintersten et al. 2004) to derive iPS cells for the generation of live chimeric mice and then tested germline transmission of the mutant allele. Fibroblasts migrating out from frozen-thawed tail snips exhibited viability, rate of growth, and morphological characteristics similar to those from fresh cultured tail snips (Fig. 1). After that, fresh tail-derived fibroblasts, 1 week frozen-thawed tailderived fibroblasts and 1 month frozen fibroblasts were transfected with retroviral vectors expressing three transcription factors (Oct3/4, Sox 2 and Klf4). At approximately 20 days after transfection of cells and growth in ES cell medium containing fetal calf serum plus leukemia inhibitory factor (LIF), multiple, morphologically discernable ES-like colonies were observed (Fig. 2).

In one $10 \mathrm{~cm}$ dish for each group, 41, 38, and 15 distinct clones were observed, picked and expanded from cells cultured from fresh tail, 1 week frozenthawed tail, and 1 month frozen-thawed fibroblasts (Table 1). These results indicate that fibroblasts obtained from frozen tail tissues are as suitable as fresh tail tissues for the generation of clonal ES celllike clonal populations of cells. While the smaller number of viable clones from 1 month frozen fibroblasts suggests that prolonged storage of intact somatic tissues at ultra-low temperatures may be detrimental and reduce the efficiency of reprogramming. Thus, while the feasibility of deriving iPS cells from frozen-thawed tissues was the priority of this study, the impact of storage conditions, such as duration and temperature of storage, is an important focus of future studies.

Identification of DsRED iPS cells Testing for ES cell-like cell surface marker
and enzymatic activity

In order to identify potential iPS cells with characteristics equivalent to ES cells, the expression of cell surface markers was examined by in vitro assays. It is well established that undifferentiated mouse ES cells express the specific cell surface epitope SSEA1 (Solter and Knowles 1987) and alkaline phosphatase activity (Wobus et al. 1984). Strong alkaline phosphatase activity was measured in all clonal cells from frozen-thawed fibroblasts and tail-derived fibroblasts that maintained ES cell-like morphological characteristics after propagating several passages in culture. There were also cells staining positive for SSEA1 (Fig. 3a, b). Further, a high percentage of cells showed both strong alkaline phosphatase activity and SSEA1 marker expression (Table 2), consistent with undifferentiated and pluripotent ES cell-like iPS cells. 

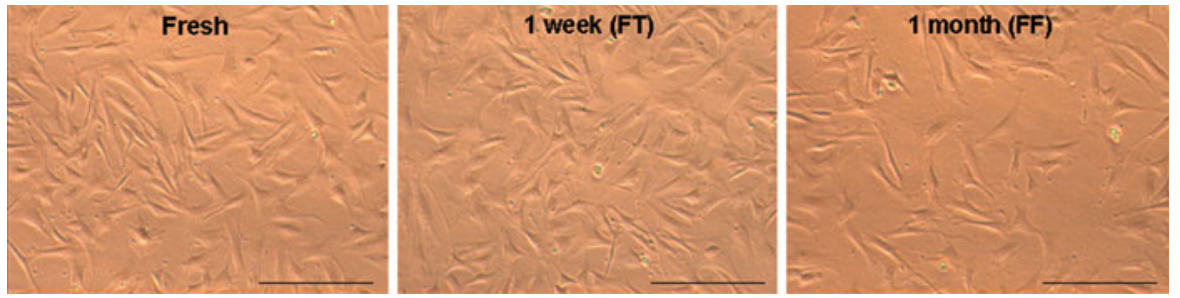

Fig. 1 Propagation of fibroblasts from mouse tail tissue. Morphology of tail fibroblasts from the culture of fresh tissue, 1 week frozen tail (FT) and 1 month frozen fibroblasts (FF). Magnification $\times 4$. Scale bar $=200 \mu \mathrm{m}$
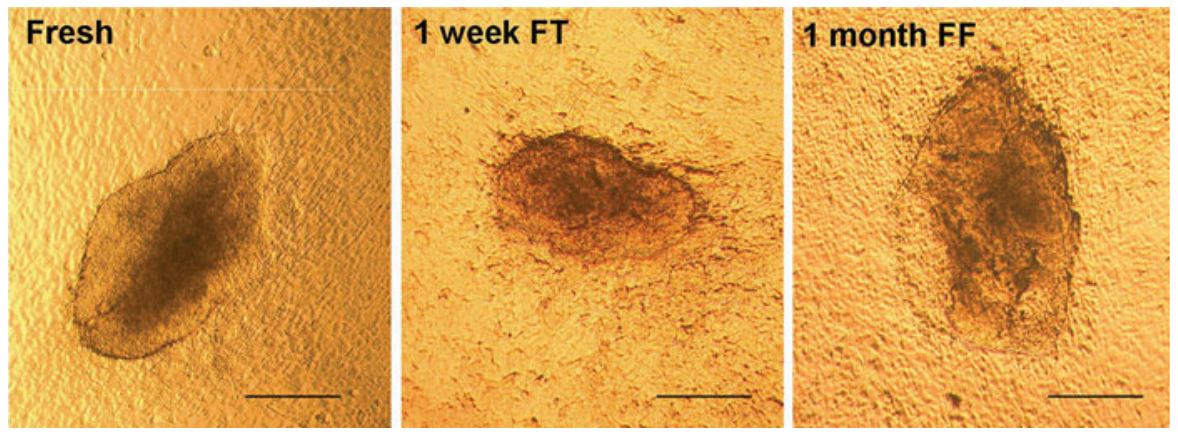

Fig. 2 iPS cell colonies formation by transfection of three transcription factors (Oct3/4, Sox 2 and Klf4). Around 20 days after transfection, fibroblasts were reprogrammed to form iPS

Table 1 iPS cells derived from freshly harvested and 1 week and 1 month frozen stored tail tissues

\begin{tabular}{ll}
\hline Source tissue & $\begin{array}{l}\text { No. of expandable iPS } \\
\text { cell clones }\end{array}$ \\
\hline Fresh tail & 41 \\
1 week frozen tail & 38 \\
1 month frozen fibroblast & 15 \\
\hline
\end{tabular}

Testing pluripotency of DSRED iPS cells and derivation of germline chimeras from DsRED iPS cells

One test to definitively confirm the pluripotency of iPS cells is to derive chimeric mice which can be analyzed for germline transmission of the mutant allele. To do this, we first assessed the chromosome count of alkaline phosphatase-positive/SSEA1 positive iPS cells from frozen-thawed tail-derived fibroblasts and frozen-thawed fibroblasts (Fig. 4a). Of these, iPS cells shown to be at least $80 \%$ diploid were microinjected into the blastocoel cavity of Balb/C derived blastocysts (8-12 cells/blastocyst) and transferred into the uterine horn of pseudopregnant CD-1 female foster mothers (Table 3). Chimeric mice were cell colonies from fresh tail tissue (Fresh), 1 week frozen tail (FT) and 1 month frozen fibroblasts (FF). Magnification $\times 4$. Scale bar $=200 \mu \mathrm{m}$

generated from both groups of iPS cells, as evidenced by expression of the transgenic red fluorescent protein (Fig. 4b). Chimeric male mice with high contribution of DsRED iPS cells (Fig. 4c) were crossed with wild-type $\mathrm{CD} 1$ females. Mice in the $\mathrm{F}_{1}$ mice showed black (C57BL/6) or agouti (C57BL/ 6;CD1) coat color, besides, they all contained the DsRED transgene when examination by PCR (Fig. 5), further confirming germline transmission of an induced, mutant allele. These results confirmed that iPS cells derived from frozen-thawed fibroblasts and tail-derived fibroblasts were pluripotent and thus able to give rise to normal mice of the expected genotype. Also, in term of number of chimeric mice generated, there seems to have insignificant difference in efficiency between the group of 1 month frozen-thawed fibroblasts and 1 week frozen-thawed tail-derived fibroblasts (Table 3).

\section{Discussion}

The results of our study have three implications. First, mouse fibroblasts derived from frozen tail tissue or 


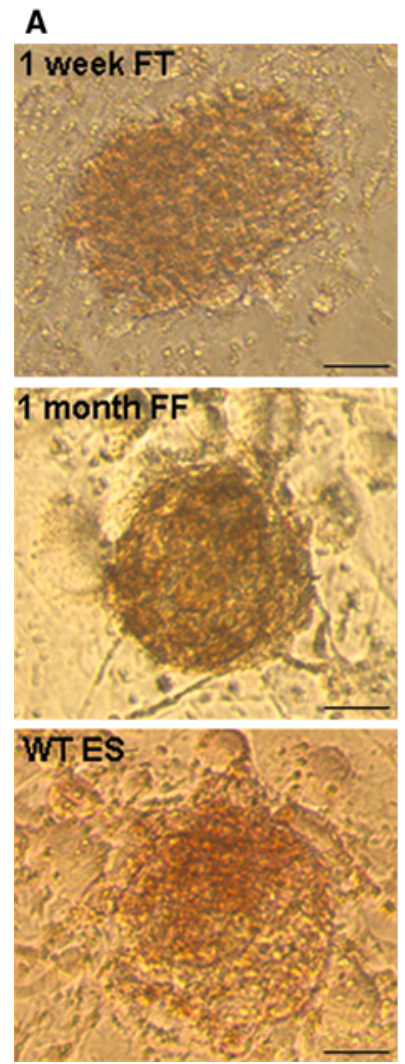

Fig. 3 Testing the identity of the iPS cell by molecular markers. The iPS cells that derived from frozen tail (FT)/ fibroblasts (FF) had strong alkaline phosphatase activity (a) and had strong expression of mouse ES cell surface marker

Table 2 Alkaline phosphatase activity and SSEA1 immunostainng for iPS cells

\begin{tabular}{lll}
\hline Source tissue & $\begin{array}{l}\text { No. of iPS } \\
\text { cell clones }\end{array}$ & $\begin{array}{l}\text { No. (\%) of iPS cell } \\
\text { clones immunopositive for } \\
\text { both AP andSSEA1 }\end{array}$ \\
\hline $\begin{array}{l}1 \text { week } \\
\text { frozen tail }\end{array}$ & 38 & $31(70.5)$ \\
$\begin{array}{l}1 \text { month frozen } \\
\text { fibroblast }\end{array}$ & 15 & $11(73.5)$ \\
\hline
\end{tabular}

frozen tail-derived fibroblasts can be successfully reprogrammed into iPS cells. Viable fibroblasts can be retrieved from frozen storage in freezing solution supplemented with DMSO. DMSO is a well known cryoprotectant, which is essential to prevent cryodamage and cell degeneration (Lovelock and Bishop 1959; Wood et al. 1993). The use of DMSO has been shown to preserve cells suitable for nuclear transfer
SSEA1 (b). They showed similar expression pattern as the C57BL/6J wild type $(\mathrm{WT}) \mathrm{ES}$ cells. Magnification $\times 20$. Scale bar in panel a and b, $50 \mu \mathrm{m}$

(Fahrudin et al. 2001). Also, three transcription factors (Sox2, Oct3/4 and Klf4) not including cmyc are sufficient for successful reprogramming of frozen fibroblasts. It has been shown that there is a direct molecular interaction between Klf4 and Oct4 and Sox 2 which is crucial for efficient somatic cell reprogramming (Wei et al. 2009). Further, reactivation of c-myc retrovirus is implicated in tumor formation in iPS cell-derived mice (Okita et al. 2007). Therefore, it was important in our study to demonstrate that iPS cells to be used to derive live mice could be generated in the absence of c-myc. Recent studies have used transposon technology to derive transgene-free iPS cells, which further reduces the risk of genetic mutation and transgene reactivation (Wang et al. 2008; Yusa et al. 2009).

Secondly, tail tissues and tail-derived fibroblasts are easy to obtain, simple to preserve, and costeffective to store frozen, thus providing a nearly 

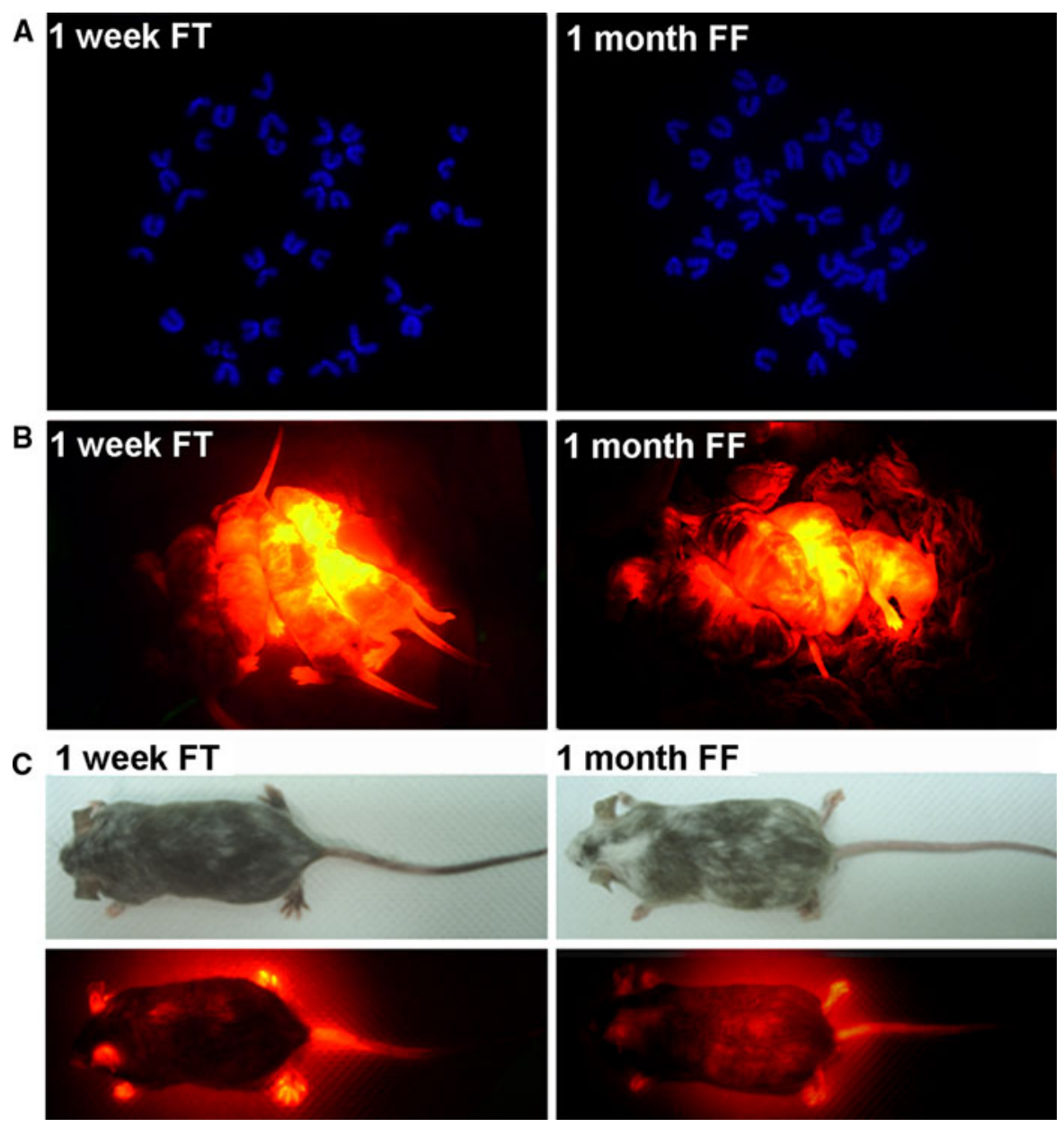

\section{1 month FF}

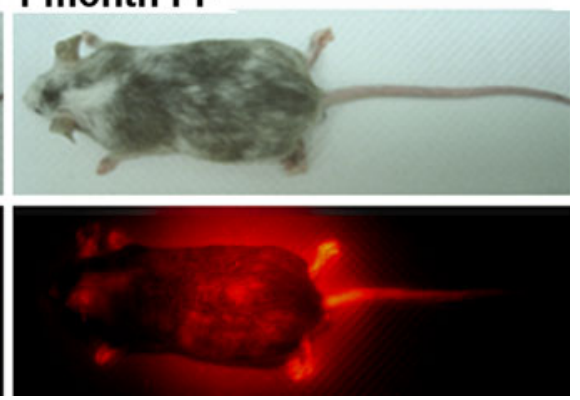

Fig. 4 DsRed iPS cells obtained from 1 week frozen tail (FT) and 1 month frozen fibroblasts (FF) were taken for blastocyst injection. a iPS clones expanded in standard ES cell medium were predominantlyeuploid. b These iPS cells were able to

generate chimeric pups with red fluorescent protein (RED) expression. c The chimeras with high chimerism and RED expression were taken for germline testing

Table 3 Summary of blastocyst injections

\begin{tabular}{llllll}
\hline Source tissue & Cell line & $\begin{array}{l}\text { No. blastocysts } \\
\text { injected }\end{array}$ & $\begin{array}{l}\text { No. chimeras } \\
(\% \text { injection) }\end{array}$ & Chimerism (\%) & $\begin{array}{l}\text { Germline } \\
\text { transmission }\end{array}$ \\
\hline 1 week frozen tail & FT-4B & 24 & $3(12.5)$ & $40-90$ & Yes \\
& FT-5B & 24 & $3(12.5)$ & $40-80$ & Yes \\
1 month frozen fibroblast & FF-2E & 31 & $4(12.9)$ & $40-90$ & Yes \\
& FF-8E & 22 & $3(13.6)$ & $10-80$ & Yes \\
\hline
\end{tabular}

unlimited source of cells for reprogramming at any time in the future. The efficiency of the process is supported by the fact that, at least for short-term storage, the number of iPS clones from frozen tail was comparable to that from fresh tail. This is likely because fibroblasts that migrate out from frozen- thawed tail divide and regenerate new fibroblasts like those from fresh tail, thus promoting efficient reprogramming. Although not directly studied, this suggests that reprogramming of frozen fibroblasts transfected soon after thawing will be less efficient than after several days in culture, as the freeze-thaw 


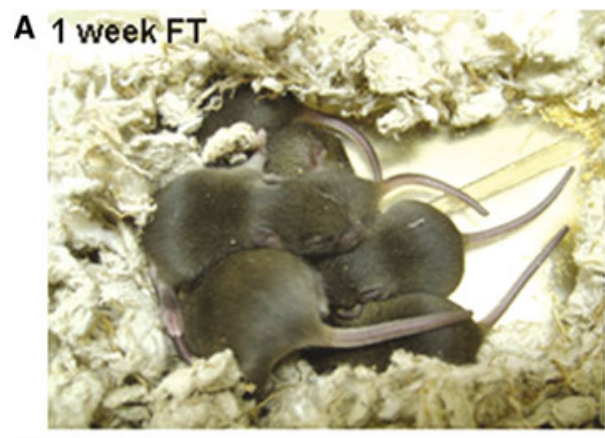

B 1 week FT

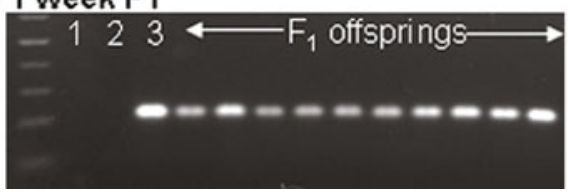

Fig. 5 Germline transmission testing by crossing the chimeras with CD1 females. a All F1 offsprings showed black or agouti color but no white color, indicating thatgermline transmission from DsRed iPS cells. b These mice were confirmed with

process will kill or disable a population of inferior or otherwise non-viable cells. However, our data suggests that reprogramming may be less efficient on frozen-thawed cells obtained from tissues after prolonged ultra-cold storage. Future studies are needed to determine the role of storage conditions on the ability to derive iPS cells from frozen tail as well as other somatic tissues.

Thirdly, our data confirms that iPS cells derived from frozen tail and tail-derived fibroblasts are sufficiently pluripotent to contribute to the germline. This is important because it supports the contention that frozen tissues and cells can be used as an alternative to ES cells and germplasm for the cryopreservation of mutant mouse lines. To our knowledge, this is the only publication demonstrating that iPS cells derived from frozen tissues and cells can be used to reanimate an entire animal, and not just a few specific cell types and/or organs. For example, in an earlier study, that cyropreserved human primary dermal fibroblasts could be reprogrammed into iPS cells, but they were subsequently differentiated only into haematopoietic progenitors (Raya et al. 2009). Our study extends these early results by demonstrating that iPS cells from frozen tissues and cells can differentiate into a variety of tissues, most importantly germ tissues (sperm) that can give rise to subsequent generations of mice.

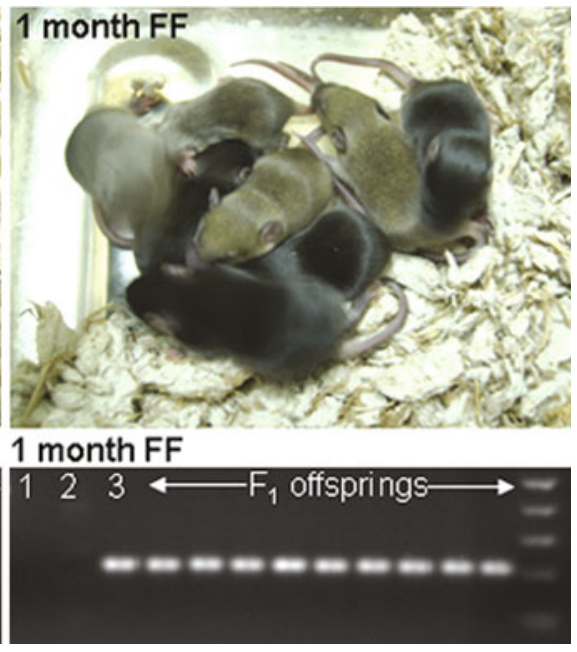

DsRed transgene by PCR genotyping. Lane 1 no DNA (negative control), lane 2 non-transgenic WT and lane 3 DsRed transgenic (positive control) showing $300 \mathrm{bp}$ PCR amplicon

Recently, viable, fully iPS cell-derived mice have been generated by tetraploid complementation of fresh cells (Kang et al. 2009; Zhao et al. 2009; Boland et al. 2009), although our results suggest that similar results could be obtained using frozen-thawed cells. Further, we demonstrated that iPS cells derived from frozen tissue and cells do not lose their pluripotency. Therefore, together these results suggest that fully cloned animals can be reconstituted from tissues and/or cells that have been frozen and maintained viable in storage.

We demonstrate here for the first time that frozen tail tissues and frozen tail-derived fibroblasts can be reprogrammed into iPS cells which retain pluripotency, contribute to the germline, and generate chimeric mice that transmit a transgene through the germline. Tissues and fibroblasts are easy to obtain from mice, and are simple, fast, and cost-effective to maintain in frozen storage. Therefore, cryopreservation of somatic tissues and/or cells is a viable method for ensuring a reliable and steady source of cells for the conversion of somatic tissues into embryos, and the reanimation of frozen stocks into live mice through generation of iPS cells.

Acknowledgments This work was supported in part by a U42 award (no. RR014905) from the National Center for Research Resources, National Institutes of Health, to establish the Mutant Mouse Regional Resource Center (MMRRC) at UC 
Davis, and by UC Davis Mouse Biology Program research funds.

Open Access This article is distributed under the terms of the Creative Commons Attribution Noncommercial License which permits any noncommercial use, distribution, and reproduction in any medium, provided the original author(s) and source are credited.

\section{References}

Aoi T, Yae K, Nakagawa M et al (2008) Generation of pluripotent stem cells from adult mouse liver and stomach cells. Science 321:699-702

Boheler KR, Czyz J, Tweedie D et al (2002) Differentiation of pluripotent embryonic stem cells into cardiomyocytes. Circ Res 91:189-201

Boland M, Hazen JL, Nazor KL et al (2009) Adult mice generated from induced pluripotent stem cells. Nature 461:91-94

Evan MJ, Kaufman MH (1981) Establishment in culture of pluripotential cells from mouse embryos. Nature 292:154-156

Fahrudin M, Otoi T, Susuki T (2001) Developmental competence of bovine embryos reconstructed by the transfer of somatic cells derived from frozen tissues. J Vet Med Sci 63:1151-1154

Kang L, Wang J, Zhang Y et al (2009) iPS cells can support full-term development of tetraploid blastocyst-complemented embryos. Cell Stem Cell 5:1-4

Lovelock JE, Bishop MWH (1959) Prevention of freezing damage to living cells by dimethyl sulphoxide. Science 183:1394-1395

Martin GR (1981) Isolation of a pluripotent cell line from early mouse embryos cultured in medium conditioned by teratocarcinoma stem cells. Proc Natl Acad Sci USA 78:7634-7638

Nakagawa M, Koyanagi M, Tanabe K et al (2007) Generation of induced pluripotent stem cells without Myc from mouse and human fibroblasts. Nat Biotechnol 26:101-106
Okita K, Ichisaka T, Yamanaka S (2007) Generation of germline-competent induced pluripotent stem cells. Nature 448:313-317

Rathjen J, Rathjen P (2001) Mouse ES cells: experimental exploitation of pluripotent differentiation potential. Curr Opin Genet Devel 11:587-594

Raya A, Rodriguen-Piza I, Guenechea G et al (2009) Diseasecorrected haematopoietic progenitors from Fanconi anaemia induced pluripotent stem cells. Nature 460:53-59

Solter D, Knowles BB (1987) Monoclonal antibody defining a stage-specific mouse embryonic antigen (SSEA-1). Proc Natl Acad Sci 75:5565-5569

Takahashi K, Yamanaka S (2006) Induction of the pluripotent stem cells from mouse embryonic and adult fibroblast cultures by defined factors. Cell 126:663-676

Vintersten K, Monetti C, Gertsenstein M et al (2004) Mouse in red: red fluorescent protein expression in mouse ES cells, embryos, and adult animals. Genesis 40:241-246

Wang W, Lin C, Lu D et al (2008) Chromosomal transposition of piggyBac in mouse embryonic stem cells. Proc Natl Acad Sci 105:9290-9295

Wei Z, Yang Y, Zhang P et al (2009) Klf4 directly interacts with Oct 4 and Sox 2 to promote reprogramming. Stem Cells 27:2969-2978

Wernig M, Meissner A, Foreman R et al (2007) In vitro reprogramming of fibroblasts into a pluripotent ES-cell-like state. Nature 448:318-324

Wernig M, Meissner A, Cassady JP et al (2008) C-Myc is dispensable for direct reprogramming of mouse fibroblasts. Cell Stem Cell 2:10-12

Wobus AM, Holzhausen H, Jakel P et al (1984) Characterization of a pluripotent stem cell line derived from a mouse embryo. Exp Cell Res 152:212-219

Wood MJ, Barros C, Candy CJ et al (1993) High rates of survival and fertilization of mouse and hamster oocytes after vitrification in dimethylsulphoxide. Biol Reprod 49:489-495

Yusa K, Rad R, Takeda J et al (2009) Generation of transgenefree induced pluripotent mouse stem cells by the piggyBac transposon. Nat Methods 6:363-369

Zhao X, Li W, Lv Z et al (2009) iPS cells produced viable mice through tetraploid complementation. Nature 461:86-90 